\title{
Der Experte als ideologieverdächtiger Laie
}

\author{
Moralische Sprachkritik durch Linguisten
}

Zusammenfassung: Es kommt immer häufiger vor, dass Wissenschaftler, darunter Linguisten, ihre disziplinäre Expertise einsetzen, um damit die Formulierung politischer Ziele oder ethische Forderungen im Dunstkreis ihres Fachgebiets zu autorisieren. Wenn Wissenschaftler Politik machen, handeln sie aber auch nicht anders als am politischen Prozess und Diskurs teilnehmende Laien: ideologisch motiviert, normativ orientiert, moralisch grundiert und teilweise emotionalisiert. An konkreten Beispielen aus aktuellen wissenschaftspolitischen Diskussionen sowie durch wissenschaftstheoretische Klärung der Rolle von Ideologie, Normativität und Ethik in der linguistischen Sprachkritik wird herausgearbeitet, dass die fachliche Expertise der Diskursteilnehmer diese nicht schon dazu berechtigt, Normen zu empfehlen und deren politische Umsetzung $\mathrm{zu}$ fordern, m. a. W. sie nicht autorisiert, genuin politisch zu agieren oder gar zu agitieren.

Abstract: Scientists and scholars, including linguists, are more and more frequently using their scholarly expertise to justify the formulation of political goals or ethical demands in the sphere of their field. When scholars attempt to do politics, however, they do not act any differently than lay people participating in the political process and discourse: ideologically motivated, oriented normatively, grounded in morals and, at times, emotionalized. Based on concrete examples from current scholarly discussions on politics and policies and clarifying the role of ideology, normativity and ethics in linguistic language criticism, it is shown that the scholarly expertise of discourse participants alone does not entitle them to endorse norms and to demand their political realization. In other words, it does not by itself authorize them to act or even agitate genuinely politically.

\footnotetext{
Frase, Michael: Lehrstuhl für Deutsche Sprachwissenschaft, DFG-Projekt Typologie und Theorie der Remotivierung (TheoRem), Universität Passau, 94030 Passau, michael.frase@uni-passau.de

Harnisch, Rüdiger: Lehrstuhl für Deutsche Sprachwissenschaft, Universität Passau, 94030 Passau, ruediger.harnisch@uni-passau.de
} 
Schlüsselwörter: Ethik, Experte, Handlungstheorie, Ideologie, Laie, Moral, Political Correctness, Sprachkritik

\section{Einleitung}

\subsection{Eine Presseschau als Problemaufriss}

Das im Titel des Beitrags angesprochene spezifisch linguistische Thema steht im Kontext des in jüngster Zeit immer kritischer gesehenen generellen Verhältnisses von „Politik und Wissenschaft“. Unter dieser Schlagzeile weist Zöllner (2020: 185) einerseits darauf hin, dass die „Politik leicht der Versuchung“ erliege, „sich eine wissenschaftliche Stellungnahme zu suchen, um vermeintliche Sachzwänge zu erzeugen.“ Anderseits warnt er davor, dass die Wissenschaftler „weder die Arbeit noch die Verantwortung der gewählten Politiker übernehmen“ sollten, und fährt fort:

Wissenschaftliche Erkenntnisse sind grundlegend und unverzichtbar für Politik, aber zur politischen Entscheidung werden sie erst im Zuge der wertgebundenen Gewichtung und Abwägung durch Politiker.

(Zöllner 2020: 185)

Mit dem Hinweis auf die wertgebundene Gewichtung kommt Ideologie ins Spiel. Zur politischen Auseinandersetzung gehört diese selbstverständlich. Aber auch „in den Adern des erkennenden Subjekts fließt Blut“ (Thiel 20201) und „Wissenschaftlerinnen und Wissenschaftler haben oft starke politische Überzeugungen“ (Kocka 2020: 125). Doch dürften „diese Einstellungen“ nicht „ungefiltert auf Fragen und Ergebnisse der wissenschaftlichen Forschung“ durchschlagen (Kocka 2020: 125). Der Experte ist also nicht grundsätzlich frei von Ideologieverdacht und ähnelt darin dem Laien, nur dass sich der Experte davon frei machen sollte, während der Laie als homo politicus das nicht braucht.

Forschungsthemenwahlen und Transferbemühungen der Wissenschaft transportieren nicht selten diese politisch-ideologische Fracht und erscheinen zuweilen als Andienungen an die aktuelle Politik. Ein jüngeres Beispiel dafür ist das vom Bund initiierte Institut für gesellschaftlichen Zusammenhalt, wofür das

1 Hier und im Folgenden wird bei Zitaten aus Tageszeitungen auf Seitenangaben verzichtet, da diese in unterschiedlichen regionalen Printausgaben desselben Tages nicht identisch sein müssen und bei Onlineversionen ohnehin keine Rolle spielen. 
Bundesministerium für Bildung und Forschung (BMBF) elf Einrichtungen ausgesucht hat. Es „soll die Forschung zu diesem Themengebiet vorantreiben“ und „mit praxisrelevanten Vorschlägen dazu beitragen, gesellschaftlichen Herausforderungen zu begegnen."2 Dieses Institut und ähnliche andere könnten für Wagner (2019) „bestenfalls genaue Beschreibungen von bestimmten Ausschnitten der sozialen Wirklichkeit“ liefern. Doch

[s]chon die Frage, ob man darin dann Probleme oder ,gesellschaftliche Herausforderungen ${ }^{3}$ erkennen will, ist keine wissenschaftliche mehr, sondern eine politische. Und auch die Entscheidung, welche Maßnahmen man dann zur Beseitigung dieses Problems ergreifen will, ist eine politische, womit man den Raum des Forschens und Fragens endgültig hinter sich lassen muss.

(Wagner 2019)

Für Wagner (2018), der darüber schon früher berichtet hatte, „drängt sich der Eindruck auf, dass niemand außen vor bleibt, solange er nur der Erwartung des BMBF genügt, den ,normativen Grundgehalt‘ des neuen Instituts anzuerkennen“. Damit kommt die für die Wissenschaft notorisch heikle Frage der Normativität ins Spiel. Wagner (2018) gibt hierzu die Auffassung eines beteiligten Soziologen wieder: „die normative Aufladung des Themas verspräche [...] ,kein normales Projekt““. Ebert (2019) beschreibt an einem anderen gesellschaftswissenschaftlichen Beispiel,

wie sehr die vorherrschende Europawissenschaft in ihren eigenen normativen Bezügen

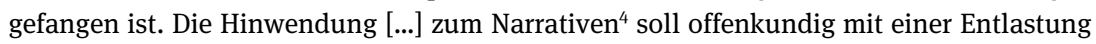
von empirischer Driftigkeit einhergehen.

(Ebert 2019)

Er sieht hierin eine nicht mehr nur normativ befangene, sondern eine ,affirmative Europawissenschaft“ im Entstehen. Von dort ist es zum Affektiven nicht weit. So spricht Thiel (2019a) in Bezug auf die o. g. Gründung des Instituts für gesellschaftlichen Zusammenhalt vom Druck der „Erfüllung wissenschaftsfremder Kriterien“ und konstatiert zu den an diesem Institut beteiligten Einrichtungen:

2 https://www.bmbf.de/de/institut-fuer-gesellschaftlichen-zusammenhalt-startet-7044.html (letzter Zugriff 09.04.2020).

3 Siehe den oben zitierten Text aus der Selbstdarstellung des BMBF, nachgewiesen in Anm. 2.

4 Die Politikwissenschaftlerin Müller-Brandeck-Bocquet, die Ebert (2019) hier zitiert, spricht von „einer neuen Großerzählung, die in turbulenten Zeiten die Richtung weisen soll.“ 
Ob diese soziale Kohäsion (wissenschaftlich) erforschen oder (politisch) fördern sollen, lässt die Namensgebung andeutungsvoll in der Schwebe. Die Folge dieser Entwicklung ist ein Klima der Unfreiheit und eine schleichende Moralisierung des wissenschaftlichen Diskurses zu Lasten genuin wissenschaftlicher Kriterien.

(Thiel 2019a)

Damit kommt zum Problem der Normativität das der Moralisierung hinzu. So diagnostiziert Thiel (2017) für die Gender Studies: „Moralische Kritik wird gegen empirische Forschung ausgespielt. Seriöse Wissenschaft wird ignoriert.“

Am Beispiel wissenschaftsjournalistischer Reaktionen auf den Vortrag von Anatol Stefanowitsch über die Dynamik von Shitstorms bei der Jahrestagung des Instituts für Deutsche Sprache 2019 sollen im Folgenden die behandelten Schlüsselbegriffe des EXPERTEN, der manchmal nicht weniger, ideologieverdächtig' ist als der LAIE, der ,wertet' und die Wissenschaft ,politisch instrumentalisiert', der ethische ,Normen' setzen will, der ,moralisiert' und ,emotionalisiert', noch einmal rekapituliert und auf die Linguistik enggeführt werden: Gerade diese Disziplin springe bereitwillig auf Versuche der Politik an, sie ,auf gesellschaftspolitische Ziele zu verpflichten“ (Thiel 2019b). Stefanowitsch habe seine Kollegen aufgerufen, „die Position des bloß analysierenden Beobachters zu verlassen und ,normativ“ zu werden“, darüber hinaus sich „für politisch korrektes Deutsch zu engagieren“ (Krischke 2019), und „unter Applaus zu einer politisch-moralischen Wende aufgerufen“ (Thiel 2019b).

[D]anach wurde des Öfteren die Emotionalität des Vortrags gelobt. Das sind bemerkenswerte Reaktionen bei einer wissenschaftlichen Veranstaltung, in der doch eher analytische Qualitäten und die Differenziertheit der Argumente zählen sollten als die herzerwärmenden Wirkungen schlichter Feindbilder und pauschalisierender Verurteilungen.

(Krischke 2019)

Dem Abdruck einer Stellungnahme des Historikers Manfred Hettling (2018) zur Erklärung des Historikerverbands gegen Rechtspopulismus und für die Einhaltung demokratischer Regeln auf der Mitgliederversammlung 2018 war von der Schlagzeilenredaktion die Bemerkung vorangestellt worden: „Die politische Resolution des Historikerverbands verkennt die Voraussetzungen, unter denen Aussagen über die Vergangenheit über das bloße Meinen hinauskommen.“ ,Meinungsstarke Wissenschaft" liegt also offensichtlich im Zeitgeist, stellt aber eine problematische, weil widersprüchliche Verbindung zwischen Objektivitätsgebot (Wissen) und seiner Aufweichung in adiecto (Meinen) dar. ${ }^{5}$

5 Dasselbe gilt übrigens auch in anderen Bereichen, etwa dem zur Objektivität verpflichteten Berichterstattungsjournalismus. Prämiert wird - im Zeitgeist liegend - eine „haltungsstarke 
Vorliegender Beitrag will den in diesem Kapitel ausgebreiteten publizistischen Befund von wissenschaftlicher (linguistischer, wissenschaftstheoretischer und philosophischer) Seite her prüfen und damit dem Gegenstand LaienWissenSprache ${ }^{6}$ das Komplement ExpertenMeinenSprache entgegensetzen.

\subsection{Gegenstand, Ziel und Methode der Untersuchung}

Der Beitrag beschäftigt sich primär mit moralischer bzw. ethischer ${ }^{7}$ Sprachkritik, die von Linguisten vorgebracht wird und die - so eine der Thesen - ideologisch geprägt ist. Dazu wird zunächst in einem hinführenden Teil der Ideologiebegriff für die nachfolgende Untersuchung präzisiert. Anschließend wird der Zusammenhang zwischen Sprachhandlungstheorie und ethischer Beurteilung skizziert, der, wie gezeigt wird, auf einem reduktionistischen AntiIntentionalismus und pragmatischen ,Perlokutionismus‘ beruht. So wird insbesondere die Betroffenenperspektive in den Mittelpunkt der Sprachkritik gerückt, was zu einer übermäßigen Vereinfachung des Sprachhandlungsmodells führt. Eine mögliche Folge, mit der sich das darauffolgende Unterkapitel auseinandersetzt, ist der Rückschluss von den Folgen einer Sprachhandlung auf Absichten und Einstellungen des Sprechers - die dieser gar nicht unbedingt verfolgt haben muss. So soll gezeigt werden, dass die handlungstheoretische Verengung unabsichtliches oder böswilliges Missverstehen ermöglicht. Ausgehend davon soll das Verhältnis von LAIE und EXPERTE im Rahmen der moralischen Sprachkritik umrissen werden: Zwar besitzen Linguisten zweifelsfrei Expertise im Diskurs über Sprache, können jedoch keine Autorität in Bezug auf ethische Fragestellungen beanspruchen. Moralische Fragen, so das Fazit des Beitrags, sollten

Berichterstattung“ (dpa/F.A.Z. 2020 über die Verleihung des Grimme-Preises an die WDRMonitor-Redaktion mit Georg Restle). In einem Essay für das WDR-Mitarbeitermagazin habe sich Restle „für einen ,werteorientierten Journalismus“ und gegen das ,Beharren auf journalistischer Objektivität““ ausgesprochen (Meier \& Hinrichs 2020: 17). „Der Journalismusprofessor Michael Haller kam in einer Untersuchung zum Migrationspakt zu dem Schluss: ,Die Tagesschau folgt ihrem tradierten Leitbild des moralisierenden Belehrungsjournalismus““ (Meier \& Hinrichs 2020: 17).

6 So der Titel der Tagung in Kiel 2019, zu der vorliegender Sammelband erscheint.

7 Die Ausdrücke moralisch und ethisch werden im fachfremden Diskurs in der Regel synonym gebraucht. Genau genommen lässt sich etymologisierend eine Differenzierung in die Praxis der (guten) Sitten (lat. mores) und die ihr zugrunde liegenden allgemeinen Prinzipien bzw. die Gesinnung (griech. Ethos) vornehmen. Diese Feinunterscheidung ist jedoch im Rahmen dieses Beitrags nicht weiter von Belang. 
besser der Disziplin überlassen werden, die sich systematisch und reflektiert mit dem richtigen Handeln beschäftigt: der philosophischen Ethik.

\section{Zum Ideologiebegriff}

Mit Tepe (2012: 15) lässt sich Ideologie als (1) „Ideen- und Wertesystem“ sowie daraus resultierend als (2) „politisches Programm“ beschreiben. ${ }^{8}$ Damit ist nichts über die (Il-)Legitimität der damit verbundenen politischen Forderungen gesagt - nach dieser Definition verfügt vielmehr jeder über eine wie auch immer geartete Ideologie. Bülow \& Herz (2014: 491-494) weisen auf eine weitere Verwendungskomponente des Ideologiebegriffs hin, wenn sie konstatieren, dass die Begriffe Ideologie und Wissenschaft eng aufeinander bezogen sind. Der Ideologievorwurf stellt demnach einen Vorwurf der Unwissenschaftlichkeit dar; gleichzeitig kann aber die Kritik an - wie auch immer gearteten - wissenschaftlichen Methoden oder Fragestellungen als ideologisch abgetan werden. Ideologisch sind damit immer nur die Anderen; die eigene Position ist stets die rational-logische (vgl. Bülow \& Herz 2014: 493). Mangels einer gemeinsamen (wissenschaftlich-begrifflichen) Grundlage wird der Diskurs so eher verhindert als konstruktiv ermöglicht (vgl. Bülow \& Herz 2014: 473-474; 508). Mit diesen Einschränkungen im Hinterkopf soll im vorliegenden Beitrag IDEOLOGIE den Wertungscharakter von Aussagen im Gegensatz zur wissenschaftlichen Wertfreiheit bezeichnen. ${ }^{9}$ Dabei geht es nicht um den Versuch, bestimmte Positionen zu delegitimieren; Ziel ist vielmehr, die „Vermischung politischer [und ethischer] Forderungen mit wissenschaftlichen Ansprüchen“ (Bülow \& Herz 2014: 508) und damit verbundene Probleme einerseits sichtbar zu machen und anderseits durch saubere Begriffstrennung zu überwinden.

\footnotetext{
8 Dies ist nur eine von vielen, teilweise einander widersprechenden Definitionen, die für den Begriff existieren und die von Tepe (2012) weiterführend behandelt werden. Schließlich verfügt der Ausdruck - nicht zuletzt aufgrund seiner marxistischen Begriffsgeschichte - über eine Bedeutungskomponente als Kampfbegriff, Unwert- oder Stigmawort (vgl. Bülow \& Herz 2014: 491-494). Diese soll aber mit der Begriffsverwendung im vorliegenden Beitrag dezidiert nicht transportiert werden.

9 Auf den Einwand, dass bereits die Wahl der Methode eine Perspektivierung und damit Wertung enthalte, ist zu entgegen, dass die Wertungen ,untersuchenswert' und ,gut' (im ethischen Sinne) qualitativ und kategorial verschieden sind. Zwar sind beide Perspektivierungen, doch ist nur eine davon ethisch wertend. Außerdem besteht der Wertfreiheitsanspruch selbst dann weiter, wenn eine vollständig neutrale Position nicht möglich sein sollte: Ziel ist keine vollständig wertfreie, sondern eine möglichst wertfreie Untersuchung.
} 
Laut Maitz (2014: 12-13) sind Ideologien im Diskurs über Sprache allgegenwärtig, aber in der Regel unbewusst, und werden nur selten explizit formuliert. Was bei Maitz für die Sprachteilnehmer gilt, trifft ebenso auf die Sprachwissenschaftler zu: Zweifelsfrei sind Forderungen nach politischer (vgl. den Untertitel von Stefanowitsch 2018: „Warum wir politisch korrekte Sprache brauchen“) und „menschliche[r] Korrektheit“ (Richter 2013; zitiert nach Tereick 2019: 396) bzw. der Beseitigung sozialer Ungleichheiten (titelgebend für Tereick 2019) als ideologisch einzustufen, da sie bestimmte Werte als gut bzw. erstrebenswert präsupponieren. Sie werden nicht nur moralisch begründet, sie setzen je spezifische Konzeptionen vom guten bzw. richtigen Handeln implizit voraus. Soziopolitische Forderungen sind jedoch - solange sie nicht selbst Untersuchungsgegenstand, etwa bei der Untersuchung von Sprachnormen, und damit selbstverständlich vollkommen legitim sind (vgl. Tereick 2019: 396) - mit dem Objektivitäts- bzw. Wertfreiheitsanspruch der Wissenschaft genuin unvereinbar. Denn normative politische Forderungen - und darum handelt es sich zweifelsfrei, wie auch Tereick (2019: 396) zugesteht - lassen sich unabhängig von ihrer Legitimität nicht uneingeschränkt aus wissenschaftlichen Erkenntnissen ableiten. In der Ethik gilt daher die unter dem Namen „Humes Gesetz“ oder „Sein-Sollen-Fehlschluss“ bekannte gängige Beschränkung, dass von deskriptiven Prämissen nicht ohne Weiteres auf normative Implikationen geschlossen werden kann (vgl. Kutschera 1999: 31-34). Darüber hinaus stellt sich die - hier nicht $\mathrm{zu}$ beantwortende - Frage, inwiefern ,das Bewusstsein für die Varianz von Lebensweisen“ (Tereick 2019: 396) überhaupt linguistisch relevant ist und ob die vorgeschlagenen (Selbst-)Regulationsmaßnahmen überhaupt den gewünschten Erfolg bringen (vgl. Jung 1996: 33f.).

\section{Sprache, Ethik und Intention}

\subsection{Sprachhandlungen und ihre Folgen als Gegenstand moralischer Sprachkritik}

Die Grundlage für sprachpolitische Forderungen bildet die Annahme, dass „Sprecher für ihre Sprachhandlungen verantwortlich gemacht werden“ (Janich 2019: 424). Mit dem Rekurs auf Moral bzw. Ethik ist in der Regel ein Universalitätsanspruch hinsichtlich der Gültigkeit der vertretenen Normen verbunden (vgl. Wimmer 1998: 47). Gleichzeitig konzentriert man sich auf die Folgen für die Betroffenen: „Im Zentrum der Aufmerksamkeit steht das betroffene Individuum“ (Tereick 2019: 386). Anders ausgedrückt, werden die Folgen relevanter 
gesetzt als die Handlung selbst bzw. als die Intention des Sprechers: „Eine Kritik an diskriminierenden Sprachhandlungen kann unabhängig von der Intention der Sprecher_in erfolgen“ (Tereick 2019: 386). Differenzierter scheint das folgende Urteil, dem zunächst zuzustimmen ist: „Was für den Sprecher funktional angemessen, weil in der Kommunikation erfolgreich ist, kann aus Sicht des Adressaten und seiner abweichenden Bedürfnisse womöglich längst nicht mehr als angemessen beurteilt werden“ (Janich 2019: 439). Die Perlokution wird in diesem Kontext jedoch als wichtiger als die Illokution angesehen. Dies wird sogar offen zugegeben: „Als deutlicher reziprokes Kriterium wird hier daher zusätzlich das der Akzeptabilität vorgeschlagen, das die Dimension der Perlokution stärkt“ (Janich 2019: 440; Herv. i. Orig.). Diese Ethik-Konzeption wird gemeinhin als Konsequentialismus bezeichnet. Der alleinige Fokus auf die Betroffenenperspektive ist jedoch aus sprechakttheoretischer Sicht unterkomplex: Sowohl Illokution als auch Perlokution sind konstitutiv. Das Verhältnis von Illokution und Perlokution soll an folgendem Beispiel aus der Alltagskommunikation illustriert werden:

(1) A: Bist du schon fertig?

B: Hetz mich nicht!

B interpretiert die Äußerung von A als Aufforderung, sich zu beeilen. Das ist eine mögliche Deutung; A kann die Aussage aber auch interrogativ als Informationsfrage gemeint haben. Betrachtet man nur die Reaktion von B, gerät diese Möglichkeit jedoch ins Hintertreffen. Die Übergewichtung der Perlokution zugunsten der Illokution kann außerdem zu Missverständnissen führen und ermöglicht nicht zuletzt das absichtliche Hineininterpretieren. So wird z. B. daraus, dass die adressierte Person beleidigt ist (Perlokution), geschlossen, dass diese Beleidigung auch die Intention des Sprechers (Illokution) war. ${ }^{10}$ Ein weite-

10 Die Typologie des Passauer DFG-Projekts TheoRem - Typologie und Theorie der Remotivierung, an dem beide Verfasser beteiligt sind und in dessen Kontext vorliegender Beitrag entstanden ist, sieht im Bereich der „gebrauchsgebundenen Remotivierung“ den Typus der Relokution vor (dazu Harnisch \& Krieger 2017: 83-85, für den appellativischen, Harnisch 2017: 262264, für den proprialen Bereich). Bei Relokution wird aus einer Äußerung die indirekte bzw. primäre Illokution getilgt und die Äußerung auf die sprachliche Oberflächenstruktur reduziert: Hast du eine Uhr dran? - Ja. Die primäre, aber nicht explizit formulierte Illokution ,Bitte sag mir die Uhrzeit‘ wird ignoriert, sodass nur auf die sekundäre Illokution - die Entscheidungsfrage, die sich aus dem reinen Wortlaut, der Lokution, erschließen lässt - reagiert wird. Beim vorliegenden Beispiel Bist du schon fertig? - Hetz mich nicht! ist im Umkehrschluss davon auszuge- 
res Beispiel: In einer Broschüre der Amadeu-Antonio-Stiftung (2015: 16), an deren Entwicklung auch linguistisches Fachpersonal beteiligt war, werden Beispiele für sog. hate speech (,Hassrede`) aufgeführt. Dort finden sich Sätze wie:

(2) Tod den Juden!

(3) Israel muss liquidiert werden,

an deren Äußerungsabsicht kein Zweifel besteht. An derselben Stelle findet sich auch das folgende Beispiel:

(4) Das Asylrecht gehört abgeschafft.

Hierbei handelt es sich laut den Verfassern der Broschüre um „[i]ndirekte Hatespeech“, die „,auf den ersten Blick“ keine „gefährliche Agitation“ sei; „[d]enken wir die Aussage jedoch konsequent zu Ende, unterstützt und legitimiert sie bestehende Gewalt“ (Amadeu-Antonio-Stiftung 2015: 16). Die zwei erstgenannten Beispiele lassen, wie erwähnt, keinen Zweifel an dieser Einschätzung; warum aber die Aussage Das Asylrecht gehört abgeschafft - zudem kontextlos - Gewalt unterstützen soll, ist gänzlich unklar. Sie kann etwa sachlich in einem juristischen Disput geäußert worden sein. Auch die Meinung, Deutschland solle keine Flüchtlinge aufnehmen, mag zwar politisch anfechtbar sein, ist aber zu keiner Zeit mit einem konkreten Gewaltaufruf gleichzusetzen. Eine solche enge Interpretation, bei der eine spezifische Illokution bestenfalls unterstellt wird, ist ungerechtfertigt und willkürlich. So kommt auch Jung (1996: 32-33) zu dem Urteil, dass „[l]eichtfertig verteilte Etiketten wie Sexist, Rassist, frauenfeindlich oder weiland Faschist“ eine „verbale Aggression“ gegen die jeweils Inkriminierten darstellen. Hier muss die Frage gestellt werden, ob diese Praxis nicht dem intendierten ethischen Anspruch diametral entgegenläuft.

Dies führt dann auch sogleich zurück zur Frage der Moral: Besonders aus moralischer Sicht macht es einen wesentlichen Unterschied, ob eine schädliche Handlung absichtsvoll geschieht oder nicht. ${ }^{11}$ Der dieser Sprachkritik zugrunde

hen, dass die Äußerung durch den Hörer mit einer anderen Illokution als der vom Sprecher intendierten angereichert wird.

11 Auf diesen Umstand weist etwa der Philosoph P. F. Strawson in seinem klassischen Aufsatz Freedom and Resentment (deutsch als Strawson 1978) hin. 
liegende Handlungsbegriff, der Folgen vor Intentionen stellt, ist aus handlungstheoretischer Sicht problematisch. Denn Intentionalität ist „das Grundmerkmal von Handlungen“ (Röska-Hardy 1997: 141). Der Handlungsbegriff impliziert „die Zuschreibung einer Absicht und anderer Einstellungen wie Überzeugungen und Wünsche“ (Röska-Hardy 1997: 141). Andernfalls würde es sich nur um (nichtabsichtliches) Verhalten handeln. Auch bei Searle (1971: 97; vgl. auch 78) findet sich dieser Gedanke im Rahmen der „Aufrichtigkeitsregel“"12 für die Indikation der illokutionären Rolle wieder: Aufrichtig kann ich etwas nur tun, wenn ich es auch absichtlich tue. Röska-Hardy (1997: 145) hat natürlich Recht, wenn sie darauf besteht, dass eine

handlungstheoretische Erklärungsweise, nach der ein einzelnes Handlungsereignis mit Hilfe der Gründe erklärt wird, die eine Person für den Vollzug der Handlung hat, nicht einfach auf sprachliche Äußerungsereignisse übertragen werden kann.

Entscheidend ist, dass beide Teile, Intention und konventionelle Bedeutung, zusammenspielen und kein Teil für sich allein ausreicht, um eine Sprachhandlung hinreichend zu beschreiben (vgl. Röska-Hardy 1997: 147). Nuyts (1997: 54) argumentiert ähnlich: Der Sprachverwender gebraucht die Sprache „willentlich und zielbewußt“, weshalb es „wesentlich [ist], die ,subjektive' Perspektive dieses Benutzers [...] einzunehmen.“ Man müsse daher in einer adäquaten Beschreibung neben dem Sachverhalt, über den gesprochen wird, dem Setting sowie den sozialen und interpersönlichen Konventionen auch die Intention des Sprechers „gegenüber dem Interaktionspartner und in bezug auf den Sachverhalt" beachten (Nuyts 1997: 55). Entscheidend ist auch hier, dass alle Faktoren notwendig sind und keiner allein hinreichend ist (vgl. Nuyts 1997: 68-69). Janich (2019: 438) ist vollkommen zuzustimmen, wenn sie schreibt, dass auch die Handlungsfolgen (pragma-)linguistisch zu untersuchen sind, da die Perlokution konstitutiver Bestandteil des Sprechaktes ist. Aber ihr Urteil gilt in beide Richtungen: Vor handlungstheoretischem Hintergrund ist es jedoch vollkommen verfehlt, einen der Faktoren perspektivisch zu stärken. Gleichzeitig legt die Verwendung bestimmter, möglicherweise konventionalisierter Formulierungen den Schluss auf bestimmte Illokutionen nahe. Wer etwa vom totalen Krieg spricht, wird sich entsprechenden ideologischen Zuschreibungen gegenübersehen, die auch mit dem rechtfertigenden Verweis auf die eigentliche Wortbedeutung nicht aus der Welt geschafft werden dürften. Die - linguistische wie ethische - Beurteilung sollte aber idealerweise fallabhängig und nicht ge-

12 Im Eng. sincerity, was teils als ,Aufrichtigkeit‘, teils als ,Ernsthaftigkeit‘ übersetzt wird. 
neralisierend erfolgen. Sprachliche Kommunikation ist und bleibt ein Prozess, bei dem die Interagierenden einander zwangsläufig Intentionen zuschreiben; und eine solche Zuschreibung kann auch revidiert werden.

Aus diesen handlungstheoretischen Überlegungen folgt weiter, dass es nur dann Sinn ergibt, Sprecher für ihre Sprachhandlungen verantwortlich zu machen (s.o.), wenn diese sich auch als eigentliche Handlungen - dies impliziert Absicht bzw. Intentionalität - einstufen lassen. Aus diesem Grund ist es auch kontraproduktiv, von der Sprecher-Illokution gänzlich zu abstrahieren. Es ist nicht nur aus (sprach)handlungstheoretischer, sondern auch aus ethischer Sicht erforderlich (und redlicher), auch die möglichen Sprecher-Illokutionen und -Intentionen im Blick zu behalten, bevor Rückschlüsse aufgrund des perlokutionären Effektes - der ja unbeabsichtigt sein könnte - gezogen werden. Zudem genügt es nicht, moralische Kriterien unreflektiert aufzustellen und ihre Durchsetzung bzw. Normierung einzufordern. Forderungen nach „Sprachkultiviertheit“ (vgl. Janich 2019: 429) oder der Akzeptanz alternativer Lebensentwürfe (vgl. Tereick 2019: 396) werden mit einer Selbstverständlichkeit vorgetragen, die sie nicht für sich beanspruchen können, und sind darüber hinaus keine rein sprachlichen, folglich auch keine genuin linguistischen Fragestellungen, da sie vor allem anhand von sozialen und moralischen Kriterien beurteilt werden. Sie berühren die Disziplin der Ethik, in der Linguisten keine nennenswerte Expertise besitzen (mehr dazu unten).

Denn die Probleme der Ethik sind vor allem Probleme der Begründung bzw. Rechtfertigung. Insbesondere die moderne Ethik ist diskursorientiert; ihr geht es darum, die Prinzipien und Maximen des ethisch richtigen Handelns gegenüber Anderen demokratisch und argumentativ durch den berühmten ,zwanglosen Zwang des besseren Arguments“ (Habermas 1982: 47; vgl. auch 52-53) herauszuarbeiten. Freilich geht es dabei um Konzepte wie FREIHEIT, GERECHTIGKEIT und WoHLERGEHEN. Die Krux besteht jedoch gerade darin $\mathrm{zu}$ bestimmen, worin diese im Einzelnen bestehen. So ist etwa die Forderung nach Diversität „[s]prachliche wie gesellschaftliche Vielfalt ist wünschenswert“ (Tereick 2014: 203) - alles andere als selbstevident, und der Verweis auf die faktische Vielfalt moderner Gesellschaften ist keine hinreichende Rechtfertigung (Sein-Sollen). Auch die Forderung nach sozialer Gerechtigkeit - „[d]ie Gesellschaft soll gerecht sein, ihre Mitglieder sollen gleichberechtigt sein“ (Tereick 2014: 203) - ist ungenügend differenziert. Natürlich soll die Gesellschaft gerecht sein - aber worin besteht Gerechtigkeit? Ist umfassende Gleichberechtigung hinreichend, um eine Gesellschaft als ,gerecht` zu qualifizieren? Und worin besteht Gleichberechtigung konkret: Chancengleichheit? Ergebnisgleichheit? Gleichbehandlung? Orientierung an individuell-subjektiven Bedürfnissen (und damit also 
doch wieder Ungleichbehandlung)? Tereick (2019: 395) bringt diese Problematik durchaus treffend auf den Punkt, liefert aber leider keine Lösung: „Was ist Ungerechtigkeit und Unterdrückung genau und wie ist diese etwa von Unfreundlichkeit und Unhöflichkeit abzugrenzen?" Ohne weitere Klärung bleiben diese Konzepte vage und subjektiv. Der Rekurs von Stefanowitsch (2018: 24) auf die ,goldene Regel' ist zwar deutlicher universalistisch und zudem nicht so stark konsequentialistisch wie die anderen skizzierten Beispiele, sondern deontologisch $^{13}$ ausgerichtet; er ist aus ethischer Sicht jedoch äußerst banal. Aus der Formel „was du nicht willst, das man dir tu, das füg“ auch keinem andern $z u^{\prime}$ lässt sich eben nicht ohne Weiteres eine allgemeingültige Regel ableiten. Das hat mit der subjektivistischen Formulierung in der 2. Person Singular $d u$ zu tun: Wodurch ist sichergestellt, dass jedes $d u$ dieselben Interessen hat? Immerhin steht die Frage nach der Allgemeingültigkeit, also der Universalitätsanspruch, der Prinzipien am Anfang ethischer Problemstellungen (vgl. Janich 2019: 425; Wimmer 1998: 47). Die Folge ist auch hier ein moralischer Subjektivismus. Problematisch ist außerdem - neben der Tatsache, dass der moralische Subjektivismus per definitionem weder universalistisch noch wertneutral ist - die theoretische Rechtfertigung. Denn einerseits ist der moralische Subjektivismus die Voraussetzung für die methodische Fokussierung der Betroffenenperspektive; gleichzeitig fungiert die Betroffenenperspektive als theoretische Rechtfertigung für den moralischen Subjektivismus.

Allen oben skizzierten sprachpolitischen Forderungen liegen jeweils spezifische Gerechtigkeitskonzeptionen - Ideologien - zu Grunde, die weder expliziert noch begründet werden. Wer dezidiert eine ethische bzw. moralische Argumentation verfolgt, muss sich dieser Problematik bewusst sein. Wahrscheinlich ist diese Aufgabe in der Ethik besser verortet als in der Linguistik.

13 Das heißt an einer Regel ausgerichtet, die bestimmt, ob eine Handlung aus sich heraus ,gut' ist. Andere Formen von Ethik sind neben Konsequentialismus und Deontologie die Tugendethik (v. a. der Antike) und die Gesinnungsethik, bei der die Intention der Handlung im Vordergrund steht. Nach Ott (2016: 7-8) - unter Verweis auf Max Weber - zeichnet sich die Gesinnungsethik, so etwa eine stark (christlich-)religiöse Moral, „dadurch aus, dass sie bestimmte moralische Grundsätze [...] rigoros vertritt“ und auch gegen mögliche negative Konsequenzen verteidigt. 


\subsection{Von der Betroffenenperspektive zur Symptomatisierung des ,Übeltäters`}

Über die in Abschnitt 3.1 angesprochenen Probleme hinaus besteht beim Fokus auf die Betroffenenperspektive die Gefahr, dass man ungerechtfertigterweise „von der Sprach- zur Sprecherkritik“ (Janich 2019: 439) am ,Übeltäter‘ gelangt. Werden dem Sprecher aufgrund der skizzierten Rückschlüsse bestimmte Haltungen, Einstellungen und Absichten zugeschrieben, kann man in Anlehnung an das Modell von Karl Bühler von ,Symptomatisierung' sprechen. Im klassischen Organonmodell von Bühler ist das Sprachzeichen neben Symbol und Signal auch „Symptom [...] kraft seiner Abhängigkeit vom Sender, dessen Innerlichkeit es ausdrückt“ (Bühler 1934: 28; Herv. i. Orig.). Symptomatisierung bedeutet analog den Rückschluss von der Verwendung eines oder mehrerer sprachlicher Zeichen auf die ,Innerlichkeit' des Sprechers. ${ }^{14}$ Damit ist mehr gemeint als nur konkrete Intentionen: Einstellungen, Überzeugungen, Wertevorstellungen. Dieser Schluss kann natürlich, wie bereits im Abschnitt über Illokutionen und Intentionen deutlich geworden sein sollte, korrekt sein, ist es aber nicht zwingend. Äußert ein unbedarfter Sprecher etwa außersprachlich vorbelastete Ausdrücke wie Arbeit macht frei, so mag man die Äußerung für ihren historischen Beigeschmack monieren können. Anzunehmen, der Sprecher sei deshalb Nationalsozialist, geht aber ohne weitere Indizien zu weit, ebenso wie damit verbundene moralische Anklagen. Solche Indizien könnten im Kound Kontext der Äußerung zu finden sein: Ist der Sprecher schon zuvor durch fremdenfeindliche Formulierungen aufgefallen? Findet die Konversation in einem politischen Rahmen statt? Ist auszuschließen, dass der Sprecher das Syntagma ironiefrei und nicht als Zitat verwendet? Diese - und sicherlich noch viele weitere - Fragen stellen sich nicht nur im Hinblick auf die Beurteilung der konkreten Kommunikation, sondern erneut auch aus ethischer Sicht. Das bereits zuvor zitierte Urteil, dass eine vorschnelle Beschuldigung und Etikettierung ebenso unmoralisch sind wie die vorgeworfenen Einstellungen, ist im Kontext der Symptomatisierung ebenso relevant. Es gilt unterm Strich, nicht nur Handlung und Folgen, sondern auch Handlung und Handelnden zu trennen. Gleichzeitig darf und soll damit keiner Apologie tatsächlichen beleidigenden oder hetzerischen Sprachgebrauchs das Wort geredet werden.

14 Vgl. dazu auch Harnisch (2018: 229-234), der ,politisch korrekte’ Ausdrücke unter dem Gesichtspunkt von „Signal und Symptom“ (229) behandelt. 


\section{4 ,Laien" und ,Experten ${ }^{6}$ in der moralischen Sprachkritik}

Bestimmt man den Laien- und Expertenbegriff anhand ihrer wissenssoziologischen Position (vgl. Bock \& Antos 2019: 61, Spitzmüller in diesem Band), so muss konstatiert werden, dass Linguisten zwar Experten im Hinblick auf Sprache, nicht jedoch im Bereich der Ethik sind und deshalb für diesen auch keine Autorität geltend machen können. Folglich kann etwa ein Sprachwissenschaftler, der selbst sprachpolitisch aktiv ist, seine Forderungen nicht ohne Weiteres linguistisch begründen. Normative Fragestellungen sind im Wissenschaftsdiskurs traditionell Gegenstand der (praktischen) Philosophie, genauer der Ethik. Moralische Kritik ist damit genuin ethisch und nicht linguistisch, weil nach ethischen, nicht linguistischen Kriterien verfahren werden muss. Zwar kann im Rahmen einer linguistischen Sprachkritik etwa die „funktionale Angemessenheit“ (Niehr 2019: 180-183) anhand ethischer Normen überprüft werden, die Linguistik bzw. die Linguisten können diese ethischen Kriterien jedoch nicht als Experten festlegen. Dass auch die Vorstellung, dass Linguisten aufgrund ihrer Profession eine herausgehobene Kompetenz in der Beurteilung und Durchsetzung von Sprachnormen hätten, eine Form von Ideologie darstellt, zeigt Maitz (2014: 19), der dies unter dem Stichpunkt des „Expertismus“ anspricht. Ebenso wie die linguistischen Laien aufgrund ihres tagtäglichen Sprechens über eine Anwenderexpertise in Bezug auf die Sprache verfügen (vgl. Harnisch 2015: 162), können sie ein alltägliches Verständnis vom ,guten Handeln‘ und ,gutem Sprechen' vorweisen - und mehr als das können auch die linguistischen Experten im Hinblick auf die Ethik nicht für sich beanspruchen. Wie Harnisch (2015: 164) am Beispiel des Anglizismen-Diskurses zeigt, sind die politischen Agitationen von Wissenschaftlern zudem überwiegend kontingent insofern, als sie eher dem Zeitgeist folgen als objektiven Gegebenheiten (wobei selbst letztere nicht ohne Weiteres politische Forderungen legitimieren, wie die Erläuterung zum „SeinSollen-Fehlschluss“ in Abschnitt 2 verdeutlicht).

Im nichtwissenschaftlichen öffentlichen Diskurs etwa über politisch korrekte Sprache hingegen werden linguistische Experten wie etwa der Anglist Anatol Stefanowitsch als Autoritäten befragt und erhalten mediale Exposition, deren politisch-normative Forderungen aufgrund ihrer sprachwissenschaftlichen Expertise legitimiert und als wissenschaftliche Fakten dargestellt werden. Wie bereits erwähnt, ist diese Expertise jedoch nur scheinbar, weil sie (bloß) linguistisch, nicht jedoch ethisch ist. Anders ausgedrückt: Die Autoritätszuschreibung (durch wen auch immer) wird von der linguistischen in die ethische Sphäre 
verschoben. ${ }^{15}$ Die Folge dieses strukturellen Ungleichgewichts ist, dass die Laien - deren politische Ansichten und Forderungen nicht weniger legitim sind - sich der Verbreitung und den teilweisen (domänenspezifischen) Normierungen zu beugen haben, wollen sie nicht als ,politisch inkorrekt‘ oder rückschrittlich wirken. Hinzu kommt, dass durch Prozesse wie die oben dargestellte Symptomatisierung prinzipiell jeder als politisch bzw. moralisch verdächtig gilt und im Diskurs gebrandmarkt wird, der sich sprachlich nicht normadäquat verhält egal, ob es sich dabei um eine bewusst provozierende Normüberschreitung oder ein unbeabsichtigtes Versehen handelt.

\section{Fazit}

Alle diese Ausführungen bedeuten nicht, dass politisch rechte oder demokratiefeindliche Diskursteilnehmer widerspruchslos toleriert werden sollen. Sie sollen aber zeigen, dass man mit der Rechtfertigung durch Wissenschaftlichkeit schnell an methodische und moralphilosophische Grenzen stößt. Fragen nach moralischen Normen und Werten erfordern ein hohes Maß an Selbstreflexivität, um Systematik und Klarheit zu wahren. Dies ist die Kernkompetenz der philosophischen Ethik, weshalb die moralische Kritik vermutlich besser in dieser Disziplin aufgehoben ist. Unabdingbar ist schließlich ein demokratischer Diskurs, der unmöglich nur auf die akademische Domäne beschränkt werden darf. Dazu gehört auch, Diskursteilnehmer nicht vorschnell durch absichtliche oder unbeabsichtigte Vorverurteilungen vom Diskurs auszuschließen. Der vorliegende Beitrag soll dazu dienen, ein stärkeres ethisch-philosophisches Problembewusstsein zu schaffen und so mehr Klarheit in den Diskurs um Moral in der Sprache zu bringen.

15 Dies ist auch im aktuellen publizistischen Zeitgeschehen in Zeiten der Corona-Pandemie zu beobachten. Dort werden nicht nur Virologen oder Epidemiologen zu den medizinischen Folgen befragt, sondern auch Psychiater, Allgemeinmediziner und Zahnärzte. Umgekehrt werden auch Virologen bisweilen als Experten zu den psychischen Folgen der Ausgangssperre befragt. Und auch Prominente wie Günther Jauch oder Boris Becker, deren Expertise sich in Grenzen halten dürfte, kommen zu Wort. 


\section{Literaturverzeichnis}

\section{Quellen}

Amadeu Antonio Stiftung (Hrsg.) (2015): „Geh sterben“. Umgang mit Hate Speech und Kommentaren im Internet. Berlin. https://www.amadeu-antonio-stiftung.de/wpcontent/uploads/2018/08/hatespeech-1.pdf (letzter Zugriff 27.02.2020).

dpa/F.A.Z. (2020): Grimme-Preis mit Haltung. Frankfurter Allgemeine Zeitung vom 4. März 2020.

Ebert, Philipp (2019): Lob für den Bürger. Europäische Normativität. Frankfurter Allgemeine Zeitung vom 8. Mai 2019.

Hettling, Manfred (2018): [Zur Resolution von Münster] Bedingungen möglicher Lektionen. Frankfurter Allgemeine Zeitung vom 31. Oktober 2018.

Kocka, Jürgen (2020): Wissenschaft macht Politik. Beschädigt sie sich damit? Forschung \& Lehre 2/2020, 124-125.

Krischke, Wolfgang (2019): Die User brauchen jetzt eine eindeutige Ansage. Emotional berührt: Die Sprachwissenschaft schlägt auf dem Kongress des Instituts für Deutsche Sprache kulturkämpferische Töne an. Frankfurter Allgemeine Zeitung vom 19. März 2019.

Meier, Christian \& Per Hinrichs (2020): [Thema] Milliarden für die Maus. Welt am Sonntag vom 23. Februar 2020, 15-18.

Thiel, Thomas (2017): Der Konformismus des Andersseins. Frankfurter Allgemeine Zeitung vom 8. November 2017.

[Thiel, Thomas] tth (2019a): Die Finanzfrage. Der ungarische Fall schärft den Blick auf deutsche Hochschulen. Frankfurter Allgemeine Zeitung vom 27. Februar 2019.

[Thiel, Thomas] tth (2019.b): Für die Freiheit. Die Wissenschaft darf sich nicht instrumentieren lassen. Frankfurter Allgemeine Zeitung vom 20. März 2019.

Thiel, Thomas (2020): Unterscheiden und herrschen. Das Wissenschaftsforum zu Köln und Essen berät über „gefährliche Wissenschaft“. Frankfurter Allgemeine Zeitung vom 5. Februar 2020.

Wagner, Gerald (2018): Mächte des Zerfalls. Der Bund lässt den sozialen Zusammenhalt erforschen. Frankfurter Allgemeine Zeitung vom 24. Oktober 2018.

Wagner, Gerald (2019): Bitte erwarten Sie erst einmal gar nichts! Liefern, liefern, liefern: Das Wissenschaftszentrum in Berlin debattiert die neuen Forderungen der Politik an die Wissenschaft. Frankfurter Allgemeine Zeitung vom 30. Oktober 2019.

Zöllner, Jürgen (2020): Politik und Wissenschaft. Forschung \& Lehre 3/2020, 185.

\section{Literatur}

Bock, Bettina M. \& Gerd Antos (2019): ,Öffentlichkeit‘ - ,Laien“ - ,Experten‘: Strukturwandel von ,Laien“ und ,Experten“ in Diskursen über ,Sprache‘. In Gerd Antos, Thomas Niehr \& Jürgen Spitzmüller (Hrsg.), Handbuch Sprache im Urteil der Öffentlichkeit, 54-79. Berlin, Boston: De Gruyter.

Bühler, Karl (1934): Sprachtheorie. Die Darstellungsfunktion der Sprache. Jena: Fischer. Ungekürzter Neudruck. Stuttgart, New York: Gustav Fischer 1982. 
Bülow, Lars \& Matthias Herz (2014): Semantische Kämpfe um Wissenschaftlichkeit und Ideologie: Gender Studies, ihre Gegner/innen und die Konsequenzen für den Sprachgebrauch und das Sprachsystem. Linguistische Berichte 240, 471-516.

Habermas, Jürgen (1982): Theorie des kommunikativen Handelns, Band 1. Handlungsrationalität und gesellschaftliche Rationalisierung. 2. Aufl. Frankfurt am Main: Suhrkamp.

Harnisch, Rüdiger (2015): Der Streit um die Anglizismen im Deutschen. Mit einem Exkurs zur Aktualität von Schmellers Schrift über die Stellung der Sprachen im internationalen Wettbewerb von 1815. Jahrbuch der Johann-Andreas-Schmeller-Gesellschaft 2013, 157-166.

Harnisch, Rüdiger (2017): Remotivierung bei Eigennamen. Kontingenz - Typologie - Theorie. Namenkundliche Informationen 109/110, 250-268.

Harnisch, Rüdiger (2018): Partizipien als meliorisierende Ersatzkonstruktionen für pejorisierte personenbezeichnende Derivata. Zu Prozessen semantischer und pragmatischer Remotivierung im Zeichen der Flüchtlings- (oder Geflüchteten-?) Krise um das Jahr 2015. In Annamária Fábián \& Igor Trost (Hrsg.), Sprachgebrauch in der Politik. Grammatische, lexikalische, pragmatische, kulturelle und dialektologische Perspektiven, 217-237. Berlin, Boston: De Gruyter.

Harnisch, Rüdiger \& Manuela Krieger (2017): Die Suche nach mehr Sinn. Lexikalischer Wandel durch Remotivierung. Jahrbuch für Germanistische Sprachgeschichte 8, 71-89.

Janich, Nina (2019): Sprache und Moral: Vom guten Sprechen als gutem Handeln. In Gerd Antos, Thomas Niehr \& Jürgen Spitzmüller (Hrsg.), Handbuch Sprache im Urteil der Öffentlichkeit, 424-446. Berlin, Boston: De Gruyter.

Jung, Matthias (1996): Von der politischen Sprachkritik zur Political Correctness - deutsche Besonderheiten und internationale Perspektiven. Sprache und Literatur in Wissenschaft und Unterricht 78, 18-37.

Kutschera, Franz von (1999): Grundlagen der Ethik. 2., völlig neu bearb. und erw. Aufl. Berlin: De Gruyter.

Maitz, Péter (2014): Kann - soll - darf die Linguistik der Öffentlichkeit geben, was die Öffentlichkeit will? In Thomas Niehr (Hrsg.), Sprachwissenschaft und Sprachkritik - Perspektiven ihrer Vermittlung, 9-37. Bremen: Hempen.

Niehr, Thomas (2019): Bewerten und Beschreiben in Sprachwissenschaft und Öffentlichkeit: Forschungsfelder und sprachtheoretische Grundlagen einer linguistischen Sprachkritik. In Gerd Antos, Thomas Niehr \& Jürgen Spitzmüller (Hrsg.), Handbuch Sprache im Urteil der Öffentlichkeit, 165-187. Berlin, Boston: De Gruyter.

Nuyts, Jan (1997): Intentionalität und Sprachfunktionen. In Gerhard Preyer, Maria Ulkan \& Alexander Ulfig (Hrsg.), Intention - Bedeutung - Kommunikation. Kognitive und handlungstheoretische Grundlagen der Sprachtheorie, 51-71. Wiesbaden: Westdeutscher Verlag.

Ott, Konrad (2016): Zuwanderung und Moral. Stuttgart: Reclam.

Röska-Hardy, Louise (1997): Sprechen, Sprache und Handeln. In Gerhard Preyer, Maria Ulkan \& Alexander Ulfig (Hrsg.), Intention - Bedeutung - Kommunikation. Kognitive und handlungstheoretische Grundlagen der Sprachtheorie, 139-158. Wiesbaden: Westdeutscher Verlag.

Searle, John R. (1971): Sprechakte. Ein sprachphilosophischer Essay. Frankfurt am Main: Suhrkamp.

Stefanowitsch, Anatol (2018): Eine Frage der Moral. Warum wir politisch korrekte Sprache brauchen. Berlin: Dudenverlag. 
Strawson, Peter Frederick (1978): Freiheit und Übelnehmen. In Ulrich Pothast (Hrsg.), Seminar: Freies Handeln und Determinismus, 201-233. Frankfurt am Main: Suhrkamp.

Tepe, Peter (2012): Ideologie. Berlin, Boston: De Gruyter.

Tereick, Jana (2014): Sick of Sicksickness! Warum stilistische Sprachkritik nicht alles ist und wie politisch-moralisch begründete Sprachkritik eine linguistische Diskurskritik informieren könnte - am Beispiel der N-Wort-Debatte in Zeitungen und Blogs. In Thomas Niehr (Hrsg.), Sprachwissenschaft und Sprachkritik - Perspektiven ihrer Vermittlung, 187-211. Bremen: Hempen.

Tereick, Jana (2019): Sprache und Diskriminierung: Soziale Ungleichheit als Gegenstand emanzipatorischer Sprachpolitik. In Gerd Antos, Thomas Niehr \& Jürgen Spitzmüller (Hrsg.), Handbuch Sprache im Urteil der Öffentlichkeit, 383-399. Berlin, Boston: De Gruyter.

Wimmer, Rainer (1998): Politische Korrektheit (political correctness). Verschärfter Umgang mit Normen im Altag. Der Deutschunterricht 50, 41-48. 